\title{
APORTES METODOLÓGICOS PARA A IMPLANTAÇÃO E AVALIAÇÃO DE EXPERIMENTOS COM SEMENTES EM RELAÇÃO A GERMINAÇÃO E AO VIGOR
}

\author{
METHODOLOGICAL SUPPORT FOR THE IMPLANTATION AND EVALUATION OF \\ EXPERIMENTS WITH SEEDS IN RELATION TO GERMINATION AND VIGOR \\ Thiago Costa Ferreira ${ }^{1}$, Manoel Rivelino Gomes de Oliveira ${ }^{2}$, Aldrin Martin Perez Marin ${ }^{3}$ \\ 1,3 Instituto Nacional do Semiárido, Campina Grande, Paraíba, Brasil - thiago.ferreira@insa.gov.br \& \\ aldrin.perez@insa.gov.br \\ 2 Universidade Federal da Bahia, Camaçari, Bahia, Brasil-manoel.oliveira@insa.gov.br
}

\begin{abstract}
RESUMO
A avaliação de sementes e plântulas em vias experimentais é uma importante fonte de conhecimentos úteis para a produção agrícola sustentável. Neste sentido este artigo tem como base a reunião e compilação de informação sobre os principais métodos de avaliação de experimentos com sementes, em relação à germinação e ao vigor. Para tal, foram enumerados pontos como a qualidade de sementes, protocolos experimentais e suas respectivas exemplificações. Assim, pode ser percebido que a utilização de outros testes e variáveis podem ser ligadas a experimentação com sementes, permitindo um uso melhor dos planos experimentais a fim de contribuir com uma melhor caracterização a este processo científico. A pesquisa com sementes pode ser melhor otimizada para que seus resultados possam ser entendidos e aplicados no ambiente agrícola com maior ênfase.
\end{abstract}

Palavras-Chave: Biometria, Experimentação Agrícola, Inovação, Tecnologia de Sementes.

\section{ABSTRACT}

The evaluation of seeds and seedlings in experimental ways is an important source of useful knowledge for sustainable agricultural production. In this sense, this article is based on the gathering and compilation of information on the main methods of evaluating experiments with seeds, in relation to germination and vigor. To this end, points such as seed quality, experimental protocols and their only examples were listed. Thus, the use of other tests and variables can be linked to experimentation with seeds, allowing a better use of experimental plans to contribute to an improvement in the characterization of this scientific process. Seed research can be better optimized so that its results can be understood and science in the agricultural environment with greater emphasis.

KEYWORDS: Biometrics, Agricultural Experimentation, Innovation, Seed Technology. 


\section{INTRODUÇÃO}

Sementes, na agricultura moderna, são ainda o principal meio de propagação de cultivos produtores de grãos, forragem, frutas, amêndoas entre outros. Sua formação dá-se na gênese denominada de fecundação, pela união de grãos de pólen com os óvulos. Esta união, realizada no interior das inflorescências, promove a formação de embriões. Como resultado deste processo, existe uma fusão de materiais genéticos, denominado de cruzamento (FERREIRA \& BORGHETTI, 2004; CARVALHO \& NAKAGAWA, 2012).

O embrião, por sua vez, é então desenvolvido no interior da inflorescência, recebendo desta os nutrientes necessários para sua posterior independência, também existe a formação de tecidos que servirão para a nutrição do embrião após o momento do desligamento da sua planta-mãe (RUDNEV, 2017; OROBINSKY et al., 2018). A independência está relacionada intimamente com o processo de germinação. Portanto, as reservas nutricionais, bem como aspectos inerentes à morfologia e genética, ligados com os fatores ambientais podem influenciar na qualidade, vigor e sanidade de sementes (CARVALHO \& NAKAGAWA, 2012).

Sendo assim, a implantação de ambientes produtivos que sejam vantajosos em vias econômicas e sociais, além de serem sustentáveis, permeia ainda pela utilização de sementes de qualidade. Levando também em consideração que estas sementes serão semeadas em locais com condições ambientais diversas e com o uso tecnologias divergentes, necessitando assim de um aporte suficiente para que os processos biológicos ocorrem de maneira satisfatória (RUDNEV, 2017; OROBINSKY et al., 2018).

Mediante estas informações apresentadas neste segmento de texto, faz-se cada vez mais necessária experimentação agrícola em relação à tecnologia de sementes, sumária para resolução de problemáticas inerentes à tecnologia de sementes (CARVALHO \& NAKAGAWA, 2012). O principal ponto ligado a experimentação em tecnologia de sementes, portanto, seria a germinação (AGARWAL, 2018; KHARE \& BHALE, 2016).

Outro importante ponto, ligado a esta referida experimentação, seria o vigor de sementes e plântulas. Nesse aspecto, tem-se a clareza da condição fenotípica dos propósitos com a demonstração de suas qualidades. Levando em consideração que um lote de sementes pode apresentar um alto percentual de germinação, mas não ser vigorosa o bastante para sobreviver em condições de campo, por exemplo (CARVALHO \& NAKAGAWA, 2012).

Métodos experimentais e pesquisas direcionadas a área de tecnologia de sementes, já referidos no início deste documento, tem sua ênfase em ações de trabalho com armazenamento, tratamento, fisiologia e tecnologia da produção em campo, entre outros importantes pontos. A mensuração de caracteres biológicos pode, por sua vez, descreverem a qualidade da germinação e o vigor de lotes de sementes, com uma miscelânea de protocolos e métodos disponível na atualidade, é fundamental nesse processo de produção de conhecimento (RUDNEV, 2017; OROBINSKY et al., 2018).

Assim sendo, com esta tão grande produção necessidade de conhecimento se faz sumária a descrição e reunião de métodos de experimentação que possam melhor serem aproveitados para a mensuração dos referidos processos biológicos inerentes a germinação e vigor (KHARE \& BHALE, 2016; AGARWAL, 2018). Para tal, a literatura descreve e reúne estes processos analíticos ainda é bastante escassa, fator este que pondera a utilização e repetição de métodos de análise em virtude de outros artigos publicados, mas sem muitas vezes que haja uma base conceitual e analítica para a realização destes testes segundo descrevem (RUDNEV, 2017; OROBINSKY et al., 2018; RIFNA et al., 2019). Sendo assim, o objetivo desta revisão foi descrever métodos para a experimentação e análise de sementes com ênfase na germinação e vigor.

\section{DESENVOLVIMENTO}

\section{Bases para a experimentação com sementes}

Em primeiro plano, têm-se a visualização dos lotes de sementes tendo em vista fatores como sua genética e o ambiente ao qual foram produzidos (RUDNEV, 2017; OROBINSKY et al., 2018). Sobre a genética, fatores como precocidade de maturação, quantidade de sementes por frutos, fatores ligados a fisiologia e morfologia são muitos importantes (KHARE \& BHALE, 2016; AGARWAL, 2018). Tais pontos são também visualizados como produtos do meio ao qual as sementes são produzidas. Ligado a estes dois pontos, podem ser referidos os seguintes artigos: Andrade et al. (2020) e Silva et al. (2020) como sendo exemplos atuais que ponderam sobre ações de fenotípicas como sendo sumárias e importantes na qualidade de sementes. Maiores informações sobre a relação acerca do 
fenótipo e a qualidade de sementes podem ser visualizadas nos escritos de Khare \& Bhale (2016) e Agarwal (2018).

Com a planta estabelecida, o auge da sua maturidade fisiológica pode ser encadeado com o processo de formação de sementes por meio da fertilização, no qual ocorre a transferência do pólen (gameta masculino), por meio de vias passivas (dispersão pelo vento, animais ou água) ou ativas (ejeção, por exemplo) para a porção feminina da inflorescência (FERREIRA \& BORGHETTI, 2004; CARVALHO \& NAKAGAWA, 2012). Neste ponto, importantes pesquisas, principalmente, ligadas ao cruzamento genético, são promovidas ao passo da necessidade de produção de variedades (KHARE \& BHALE, 2016; AGARWAL, 2018).

De maneira correta, tais sementes devem ser manejadas corretamente em virtude da colheita, armazenamento e beneficiamento (CARVALHO \& NAKAGAWA, 2012). A maturação ocorre com o acúmulo de água, sais minerais e compostos orgânicos (KHARE \& BHALE, 2016; AGARWAL, 2018). Maiores informações sobre as condições de formação das sementes, diferenciação e maturação de tecidos e aportes fisiológicos podem ser mais bem esclarecidas nos escritos de Rudnev (2017), Orobinsky et al. (2018), Rifna et al. (2019) e Daibes \& Cardoso (2020).

A composição química das sementes também é um outro importante ponto ligado à experimentação com sementes na atualidade (KHARE \& BHALE, 2016; AGARWAL, 2018). Segundo Carvalho \& Nakagawa (2012), este é um fator importante a ser entendido em relação às sementes e, também, em paralelo com os grãos, usados para diversos fins. A pesquisa em relação a este ponto leva em consideração os pontos descritos no parágrafo anterior e, atualmente, tem sido mensurada por meio de protocolos bioquímicos, como descrevem Wijewardana et al. (2019) e Mrabet et al. (2020).

Uma vez desenvolvida, a semente precisa ser colhida. Este processo abre um leque de trabalhos ligados a diversos ramos das engenharias principalmente. Pela necessidade de produção de maquinários para colheita e beneficiamento. Pois, conforme descrevem Carvalho \& Nakagawa (2012), a produção de sementes deve ser bem planejada, beneficiando toda uma cadeia produtiva. Artigos atuais que podem servir de base conceitual para trabalhos nesta etapa são os seguintes: Rudnev (2017) e Orobinsky et al. (2018).

Os resultados positivos obtidos em todos os passos assinalados nos parágrafos anteriores podem ser acrescidos com a perspectiva do tratamento de sementes. Tal ação compreende na veiculação de substâncias ou energias nas sementes, de modo a diminuir a ação de pragas e, também, em alguns casos, possibilitando melhorias na morfologia e na fisiologia das plantas, segundo afirmam Machado (2000), Rifna et al. (2019) e Daibes \& Cardoso (2020). Na atualidade, além de compostos sintéticos, têm sido utilizados artifícios como materiais inertes, produtos orgânicos e energia (RIFNA et al., 2019). Maiores informações sobre métodos de tratamento de sementes podem ser visualizadas nos escritos de Machado (2000) e Rifna et al. (2019). Este ponto tem sido também ligado à proposição da sanidade de sementes (KHARE \& BHALE, 2016; AGARWAL, 2018).

Logicamente, estas sementes podem também ser armazenadas antes do seu uso, fator importante para a conservação e consolidação de áreas de agricultura sustentável (KHARE \& BHALE, 2016; AGARWAL, 2018). Neste processo, segundo descrevem Carvalho \& Nakagawa (2012) a utilização de artifícios ambientais de controle de umidade e temperatura, principalmente, são importantes para a longevidade de sementes.

\section{Requerimentos importantes para a montagem de ensaios com sementes}

Em virtude da utilização das tecnologias elencadas no tópico anterior, os produtos destes experimentos são lotes de sementes passíveis de serem analisados por meio de variáveis e técnicas analíticas (KHARE \& BHALE, 2016; AGARWAL, 2018). Para a análise destas sementes são utilizadas tecnologias de montagem de ensaios, que em sua maioria, permitem a veiculação de sementes à ambientes que sejam propícios de alguma maneira a promoção da germinação de acordo com os escritos de Rudnev (2017), Orobinsky et al. (2018), Rifna et al. (2019), Daibes \& Cardoso (2020), Wijewardana et al. (2019) e Mrabet et al. (2020).

Nesse sentido, ainda sobre as questões experimentais, podem ser elencados, segundo Carvalho \& Nakagawa (2012), os seguintes pontos a serem observados em relação a produção da pesquisa em tecnologia de sementes:

- Luz: nesse quesito, segundo está descrito no Brasil (2009), existem sementes que germinam somente no escuro ou no claro, também aquelas que não existem restrições. Também existem artigos que descrevem a germinação de acordo com a incidência de frações de luz 
(cores). No geral a medida da luz pode ser feita em relação ao período de exposição e/ou a intensidade de luminosidade;

- $\quad$ Temperatura: de acordo com Brasil (2009) e Brasil (2013) espécies vegetais apresentam temperaturas ótimas para seu desenvolvimento, algumas delas são fixas e outras são faixas, em relação à análise em ambientes controlados. Estas temperaturas podem também estar ligadas ao ambiente externo e, portanto, a sua variação de temperaturas;

- Substratos: Na Regra de Análises de Sementes (RAS) (BRASIL, 2009), em relação ao local onde as sementes serão colocadas para germinar, tem-se descrito o emprego de papel de germinação, areia ou vermiculita; em ambos os substratos são usadas as localizações sobre e entre (dentro do substrato), além do rolo de papel. Maiores detalhamentos podem ser visualizados nos escritos em Brasil (2009) e Brasil (2013). Também podem ser usados substratos diversos como tratamentos para a visualizar a germinação ou emergência em condições diferenciadas. Maiores informações sobre a fisiologia de sementes, em relação às necessidades da germinação, podem ser encontradas nos escritos de Benech-Arnold (2004).

Faz-se o friso que técnicas de quebra de dormência também podem ser empregadas, de acordo com a literatura, para melhorar a germinação, em termos de quantidade e agilidade, segundo os escritos de Machado (2000) e Rifna et al. (2019). Faz-se necessário observar principalmente, como descrevem os escritos de Brasil (2009) e Brasil (2013), que para melhor entendimento sobre tais parâmetros pesquisas devem ser realizadas para melhorar o entendimento destas necessidades e isto, por si só, como descrevem Rifna et al. (2019), pode ser ligado a uma experimentação. Mediante todos estes pontos elencados, deve-se obedecer a um delineamento estatístico a ser usado para a montagem e avaliação dos ensaios, conforme descrevem Rudnev (2017), Orobinsky et al. (2018), Rifna et al. (2019), Daibes \& Cardoso (2020), Wijewardana et al. (2019) e Mrabet et al. (2020). Métodos estatísticos em análise de sementes serão esclarecidos no tópico a seguir.

\section{Métodos estatísticos mais usados na experimentação em tecnologia de sementes}

Em relação à análise estatística a ser usada em ensaios de tecnologia de sementes, de maneira sucinta, são necessários que sejam observados em primeiro plano os seguintes pontos, elencados por Banzato \& Kronka (2006):
- $\quad$ Experimento ou ensaio: trabalho planejado com o intuito de comparação entre tratamentos ou populações;

- Tratamento ou amostra: método, elemento ou amostra populacional com características uniformes;

- Delineamento experimental: plano de distribuição das unidades experimentais;

- Hipóteses: Se faz determinada afirmação sobre uma população, usualmente sobre um parâmetro dessa, desejamos saber se os resultados experimentais provenientes de uma amostra contrariam ou não tal afirmação.- HO hipótese nula, que está sob teste e H1 é a hipótese alternativa que é aceita como verdadeira sendo HO falsa;

- $\quad$ Casualização: a coleta dos dados ou a disposição dos tratamentos deve ser casualizadas ou aleatório;

- Repetições: deve haver repetições nos tratamentos, comumente com trabalho em sementes têm sido usadas e descritas na literatura a proposição de 400 sementes para caracterizar lotes (BRASIL, 2009; BRASIL, 2013), duzentas sementes em experimentos com sementes agrícolas e cem com espécies florestais, segundo pode ser observado nos escritos de Rudnev (2017), Rifna et al. (2019) e Mrabet et al. (2020). Também deve ser entendido que as amostras devem ser representativas para que seja validada a hipótese, segundo Banzato \& Kronka (2006).

Uma vez ajustados todos os pontos elencados anteriormente, pode-se ligar os experimentos a um delineamento experimental de acordo com as suas propriedades: havendo total controle das variáveis ambientais (delineamento inteiramente casualizado) geralmente usado em laboratório e não havendo controle das variáveis ambientais (delineamento em blocos ao acaso) geralmente usado em ensaios de campo (BANZATO \& KRONKA, 2006). Exemplos da utilização experimental desses delineamentos podem ser visualizados nos escritos de Rudnev (2017), Orobinsky et al. (2018), Rifna et al. (2019) e Daibes \& Cardoso (2020).

Outro delineamento importante, a utilização de esquemas fatoriais é bastante útil na experimentação com sementes, segundo Banzato \& Kronka (2006). Neste processo que são elencados podem ser comparados tratamentos de fatores diferentes, como por exemplo, tempo de armazenamento versus tratamento com produtos sintéticos. Este tipo de avaliação permite o acesso aos delineamentos acima descritos. Exemplos da utilização deste esquema experimental podem ser visualizados nos escritos de Pinto et al. (2018), Abati et al. 
(2020), Alves et al. (2020), Bazzo et al. (2020) e Carvalho et al. (2020).

Em termos de estatística paramétrica, tem-se usado com maior frequência a ANOVA, que por meio de cálculos relacionados com testes de significância podem ser utilizados parâmetros para medir a presença de diferenças estatísticas entre os lotes ou tratamentos, conforme descreve Banzato \& Kronka (2006). Maiores informações sobre esta utilização podem ser visualizadas nos escritos de Banzato \& Kronka (2006), Rudnev (2017), Orobinsky et al. (2018), Rifna et al. (2019) e Daibes \& Cardoso (2020).

Transformações de valores têm sido também utilizados em ensaios com sementes para que a normalidade dos dados seja atendida. Tal procedimento é utilizado em MLG, descrito mais adiante. Em geral, usa-se as funções arcoseno raiz de $x / 100$ e raiz quadrada de $x+1$, segundo os parâmetros descritos por Banzato \& Kronka (2006), são usadas para normalizar os dados em meio a análises paramétricas.

Também podem ser utilizados testes relacionados com análise de Regressão, que em poucas palavras, seria a colocação dos resultados de um delineamento experimental em uma equação, conforme a natureza desses dados, de acordo com as especificações descritas Por Banzato \& Kronka (2006) e exemplificadas por Souza et al. (2005), Alves et al. (2020), Morais et al. (2020) e Pinheiro et al. (2020).

Outro importante ponto, descrito na atualidade seria a utilização de Modelos Lineares Generalizados (MLGs) propostos Nelder \& Wedderburn (1972) que estatisticamente se trata da extensão dos modelos lineares normais. Pois para muitos autores, a utilização de métodos como a ANOVA e as transformações de dados podem não ser tão bem ajustados aos resultados obtidos em testes com sementes, informações descritas por Carvalho, Santana \& Araújo (2018) e Ribeiro-Oliveira et al. (2018). Aos quais ponderam diversos pontos a serem esclarecidos para que seja realizada uma análise desta natureza, como referência para tal análise os escritos de Santana et al. (2018) podem ser citados.

Vale ser salientado, como sempre é descrito, que a montagem de uma pesquisa deve ser realizada em conjunto com um estatístico para que possíveis problemas de delineamento, amostragem e afins sejam sanados antes da montagem do ensaio. Prevenindo, assim, a solução de prováveis problemas. As informações descritas neste segmento são sumarizadas de acordo com os métodos mais usados e descritos na literatura na atualidade, em virtude dos trabalhos em tecnologia de sementes. Outros percursos estatísticos podem ser utilizados, com a observação da pertinência e possibilidade estatística de cada processo experimental.

Logo, os ensaios são passíveis de serem analisados por meio de métodos que atestem fatores biológicos por meio de contagens e analogias. A seguir, serão descritos alguns dos métodos mais importantes para a análise de experimentos com sementes e plântulas na atualidade.

\section{Métodos analíticos para a análise de experimentos}

A seguir, serão descritas variáveis que podem ser usadas para a avaliação de ensaios com sementes, em relação a germinação e vigor. Tais variáveis foram compiladas dos escritos de Rudnev (2017), Orobinsky et al. (2018), Rifna et al. (2019), Daibes \& Cardoso (2020), Wijewardana et al. (2019) e Mrabet et al. (2020).

a. Germinação: mensurado após um período posterior ao semeio, pode ser usado para descrever a qualidade de lotes de sementes, também a resultados de tratamentos diversos (agentes químicos, biológicos ou físicos) em um mesmo lote entre outros (Scott et al., 1984). Sua fórmula é simplificada está descrita a seguir:

\section{$G=\frac{\text { No de sementes germinadas }}{\text { No de sementes semeadas }} x 100 \quad(I)$}

Esta variável pode ser analisada sobre a forma de uma primeira contagem e de germinação. Estas ponderam uma primeira contagem realizada por volta da metade do período de análise da germinação e no final, respectivamente. Para cada cultura comercial existe um período de avaliação estabelecido na RAS (BRASIL, 2009), algumas espécies florestais ocorrentes no Brasil também são descritas no Manual de Análise de Sementes Florestais (2013), faz-se o friso que as espécies não registradas neste manual, segundo as informações descritas no mesmo, devem ser realizadas com o uso de informações de espécies vegetais semelhantes.

É necessário ser descrito que este teste pouco serve para a visualização da dispersão de germinação em função de um espaço de tempo. Também que a sua ação de visualização ocorre após a protrusão do tegumento da semente pelos tecidos apicais do embrião em crescimento (BRASIL, 2009). 
Como exemplificação, os escritos de Daibes \& Cardoso (2020) descrevem que podem ser utilizados estes parâmetros para a visualização do comportamento de sementes de Peltophorum dubium (Spreng.) Taub. em relação a potenciais osmóticos.

b. Percentagem de plântulas normais e anormais: segundo Brasil (2009) plântulas podem ser classificadas como normais e anormais, pela visualização da morfologia externa dos tecidos em formação. A proposição é descrita em virtude da possibilidade de anormalidades no crescimento e desenvolvimento das plântulas ocasionados por fatores externos e internos à semente. Como sugestão, a visualização das informações contidas na Regra de Análises de Sementes (BRASIL, 2009), Manual de Análise de Sementes Florestais (BRASIL, 2013) e outros materiais sobre a morfologia de plântulas podem ser visualizados a fim de construir conhecimento sobre estas e poder servir de base para a avaliação do processo de classificação. Suas fórmulas estão descritas a seguir:

$$
\begin{aligned}
& P N=\frac{\text { No de plâtulas nomais }}{\text { No de plâtulas }} \times 100 \\
& P A=\frac{\text { No de plâtulas anomais }}{\text { No de plâtulas }} \times 100
\end{aligned}
$$

c. Percentagem de sementes mortas: segundo Brasil (2009), sementes mortas podem ser visualizadas como apodrecidas, não germinadas que se desfazem ao toque ou que seus embriões estão mortos. Sua fórmula de análise pode ser visualizada a seguir

$$
S M=\frac{\text { No de sementes mortas }}{\text { No de sementes semeadas }} \times 100
$$

d. Percentagem de sementes duras: segundo Brasil (2009 b), sementes duras não absorveram a quantidade de água necessária para progredirem a estágios avançados da germinação. Sua fórmula de análise pode ser visualizada a seguir

$$
S D=\frac{\text { No de sementes duras }}{\text { No de sementes semeadas }} x 100
$$

e. Índices de Velocidade: este parâmetro, segundo Scott et al. (1984), pode ser útil para a visualização da proporção de germinação (IVG) e emergências (IVE) ocorrentes em um dado espaço de tempo. Confluindo para um valor que permite a avaliação contínua das sementes em questão. Deve ser realizado em um período hábil, com a contagem das sementes germinadas em espaços de tempo pré-determinados, segundo Scott et al. (1984). Para sementes agrícolas como soja, milho, feijão e outras de rápida germinação recomenda-se a visualização diária (CARVALHO \& NAKAGAWA, 2012). Algumas sementes de espécies florestais podem ser avaliadas de maneira mais dilatada, haja vista a sua disparidade de germinação ou emergência, segundo afirmam Daibes \& Cardoso (2020). Este teste pode ser usado em condições de campo, em substrato, com a avaliação denominada de emergência. Também, pode ser descrito como um método de avaliação de vigorar fórmula para o cálculo é a seguinte:

$$
I V=\frac{\mathrm{N} 1}{\mathrm{D} 1}+\frac{\mathrm{N} 2}{D 2}+\ldots .+\frac{\mathrm{Nn}}{\mathrm{Dn}}
$$

Onde $\mathrm{N}$ = número de plântulas em cada dia da contagem; $\mathrm{D}=$ número de dias para o semeio.

f. Índice de Tempo Médio: nesse quesito, pode ser relatada, segundo Scott e colaboradores (1984), o tempo médio para germinação ou emergência das sementes, também se trata de um teste que propõe ações de vigor. Para tal verificação, pode ser usada a seguinte equação:

Îndice de Tempo Médio $=\Sigma \frac{\text { nĩti }}{\text { ni }}$

Onde: $\mathrm{ni}=$ número de sementes germinadas por dia; $\mathrm{ti}=$ tempo de incubação; i = $1 \rightarrow \mathrm{X}$ dias de germinação.

g. Índice de Resposta Média: Scott et al. (1984) descreve esta avaliação, denominada com outras nomenclaturas, como sendo uma resposta ao tempo que ocorreu $50 \%$ da germinação ou emergência. Usando uma analogia de distribuição normal, principalmente para sementes com condição de germinação desigual. Tal análise pode ser realizada em função da utilização experimental de blocos ao acaso, permitindo a observação do comportamento dos tratamentos nos blocos e a visualização de fórmulas ecológicas populacionais. A seguir observe a notação para tal quesito:

Îndice de Resposta Média $=\Sigma r^{2}-3 b *$ $(t+1) * \frac{12}{b t v(t+1)}$ 
Onde: $\mathrm{t}=$ graus de liberdade dos blocos, $\mathrm{b}=$ número de blocos e $r$ = número de germinadas em cada dia.

h. Curvas de Acúmulo de Germinação: as curvas de acumulação de médias de germinação podem ser visualizadas por meio dos modelos de distribuição normal e exponencial, maiores informações sobre tais distribuições podem ser mais bem visualizadas nos escritos de Ahsanullah et al. (2014) e Mahdavi \& Kundu (2017). Não sendo ajustados os valores nos pré-requisitos destes modelos pode ser utilizada a negativa exponencial, ou mononuclear, com o seguinte modelo

Curva Mononuclear $=1-A \exp [-k(t-t 0)]$
Curva Mononuclear $=1-A \exp [-k(t-t 0)]$

Onde: A - representa a percentagem final de germinação, TO - tempo inicial da germinação, $\mathrm{T}$ - tempo final da germinação e k - taxa de germinação.

i. Índice de Velocidade Média: nesta proposição, a germinação ou a emergência, a utilização do valor do valor de tempo médio é estabilizada na proposição da velocidade ao qual este ponto ocorreu. Usando para tal, a equação a seguir:

Îndice de Velocidade Média $=\frac{1}{\text { fndice de Tempo Médio }}(\mathrm{XII})$

j. $\quad$ Fitomassa: este processo pode ser relacionado como um método de avaliação de vigor. Assim, as plântulas podem ser pesadas ao passo que forem retiradas do local de germinação e/ou após secas em estufa $\left(60^{\circ} \mathrm{C}\right.$ até a estabilização do peso seco). Tal processo pode ser relacionado com a totalidade das plântulas presentes no final da germinação, em sua totalidade ou parcialidade corporal. Sua notação pode ser em g/plant ${ }^{\mathrm{a}-1}$. Como representação, pode ser visualizada a seguinte equação:

\section{Fitomassa $=\frac{\text { Fitomassa }}{\text { Número de plâtulas germinadas }}$}

\section{Análises de sobrevivência}

Nesse método estatístico, a sobrevivência é descrita em relação a um evento temporal e mensurada com o número, ou percentagem, de plântulas ou sementes vivas em relação a uma análise de lote ou tratamento. De modo geral, podem ser levados em consideração fatores como a população inicial (pi) e final (pf) em relação a um período, ou a gradações de temperatura, por exemplo, com o uso de um coeficiente (h). Uma importante utilização está descrita em Scott et al. (1984) e exemplificada a seguir:

Sobrevivência $=\frac{\mathrm{Pf}-\mathrm{Pi}}{\mathrm{h}}$

Equações logísticas ou glomerais tem também sido descritas como eficazes para a avaliação em sementes, maiores informações sobre estes métodos podem ser encontradas em Scott et al. (1984). Como exemplificação, pode ser descrito o resultado do trabalho "Germination and seed ecology of Buchenavia tomentosa Eichler (Combrataceae)" de autoria de Correa et al. (2020), neste artigo os autores descrevem a sobrevivência (germeabilidade) de sementes da referida espécie em função de um largo gradiente de temperaturas fixas, um bom exemplo de aplicabilidade da sobrevivência com o uso de duas outras equações, são elas:

$$
\begin{gathered}
\text { Tsub }=(\mathrm{T}-\mathrm{Tb}) \mathrm{t} 50(\mathrm{XV}) \\
\text { Tsupra }=(T \max -T)+50(\mathrm{XVI})
\end{gathered}
$$

Onde: Tsub seria o tempo termal para a germinação em termo germinaram sub-otimos, T seria a temperatura de germinação, Tmax a temperatura máxima, t50 o período em que $50 \%$ das sementes germinaram e tb por uso modelo linear.

A seguir, ainda segundo os escritos de Corrêa et al. (2020) pode ser vista que entre 10 e $30^{\circ} \mathrm{C}$ existe uma ascendência da quantidade de sementes germinadas e em seguida, no espaço entre 30 e $45^{\circ} \mathrm{C}$, existe um acentuado decréscimo da germinação, segundo descrito na figura a seguir.

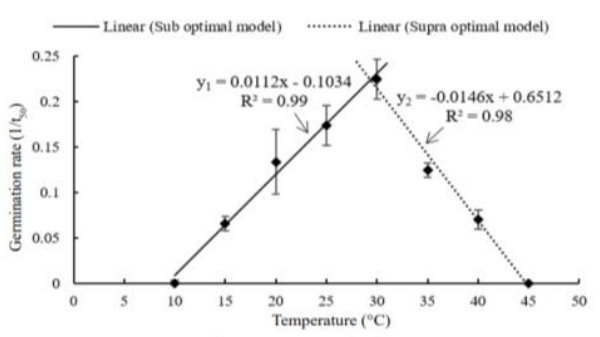

Figura 1. Germinação de Buchenavia tomentosa em relação a diferentes temperaturas segundo os escritos de Corrêa et al. (2020). 


\section{Análises de vigor}

Um importante ponto, ligado aos itens assinalados em seç̧ões anteriores seria a implantação do teste de germinação com o uso do pressuposto dos testes de vigor, propriamente ditos. Alguns autores descrevem que a análise de vigor também é realizada por meio de variáveis descritas nos itens anteriores, cabe ao usuário destas informações agrupar os separar as informações obtidas em sua pesquisa, conforme as especificações destas neste artigo ou na literatura referenciada no mesmo.

Dentre estes, o envelhecimento acelerado pode ser citado. Consistindo basicamente na exposição de sementes a condições de saturação de umidade (vapor) em um recipiente fechado a uma temperatura maior que $40^{\circ} \mathrm{C}$, por um período variante (BRASIL, 2009). Esse método viabiliza a proposição de uma qualidade ruim nas sementes, em que tratamentos ou lotes podem ser submetidos para averiguar a sua germinação e vigor em condições futuras, envelhecidas. Maiores informações sobre estes pontos podem ser visualizadas nos escritos de Gazolla et al. (2015), Bem Junior et al. (2020) e Oliveira et al. (2020).

Também, semelhantemente ao teste de envelhecimento acelerado, o teste de germinação a frio, ou teste frio, pode ser usado para que seja visualizada a germinação após a exposição a condições diferenciadas (Brasil, 2009). Nesse sentido, as sementes provenientes de lotes ou tratamentos diferentes são acondicionadas em papel de germinação e expostas a temperaturas baixas, após isto são postas em germinadores. Transcorrendo as análises descritas no item anterior, conforme descrevem Castan et al. (2018), Soares et al. (2019) e Gu et al. (2019).

A análise de tetrazólio, descrita na Regra de Análises de Sementes (BRASIL, 2009), é um teste rápido em que pode ser visualizada a coloração de tecidos vegetais vivos nas sementes, em decorrência da reação de redução do sal de Tetrazólio, pela atividade de enzimas desidrogenases envolvidas na atividade respiratória. Após a reação, podem ser distinguidos tecidos com maior ou menor respiração e, a partir daí, pode ser avaliada a viabilidade da semente e a sua capacidade de produzir uma plântula normal. Tal teste é realizado com a seguinte proposição metodológica:

- Imersão em água para que a semente esteja túrgida, fator dependente de uma maior interação com informações sobre a curva de embebição das sementes, com a entrada da água nos tecidos da semente e uma posterior facilitação da retirada do tegumento;

Seguida de uma imersão na solução de tetrazólio;
- $\quad$ Terminada por meio da visualização da coloração dos tecidos, por meio de modelos descritos na Regra de Análises de Sementes (BRASIL, 2009) e em outras literaturas.

Para a realização deste teste devem ser observados fatores como a morfologia e embebição das sementes, fatores sumários, e que sempre que possível devem ser testados os fatores deste processo (embebeciam em água, dissecação dos tecidos, embebição em solução de tetrazólio e a leitura). Deve sempre ser procurado na literatura alguma pesquisa ou informação sobre as formas de análise neste teste, existem para muitas espécies protocolos prontos que podem ser usados para tal. Exemplos diversos podem ser citados, com protocolos para espécies anuais e perenes, alguns destes podem ser visualizados nos escritos de Belniaki et al. (2020), Medeiros et al. (2020) e Mercado et al. (2020).

Outro importante teste, seria a utilização da medição da condutividade elétrica, que consiste na leitura da quantidade de sólidos dispersos das sementes em uma solução aquosa (BRASIL, 2009). A avaliação pode ser referida em percentagem de sementes viáveis e não viáveis. Períodos de embebição e métodos de leitura podem ser visualizados com maior exatidão nos escritos de Prado et al. (2019), e Krzyzanowski et al. (2019) e Medeiros et al. (2020).

\section{CONCLUSÕES}

A melhoria da qualidade da avaliação em experimentos pode ser uma importante fonte de informações, principalmente com a utilização de variáveis e métodos coerentes e ajustados a cada plano experimental, em virtude da hipótese a ser investigada. Portanto, a pesquisa com sementes pode ser mais bem otimizada para que seus resultados possam ser melhores entendidos e, assim, aplicados no ambiente produtivo.

\section{AGRADECIMENTOS}

Agradecemos ao Instituto Nacional do Semiárido pela disponibilização da bolsa do primeiro autor.

\section{REFERÊNCIAS}

ABATI, J. et al. Physiological response of soybean seeds to spray volumes of industrial chemical treatment and storage in different environments. Journal of Seed Science, v.42, e202042002, 2020. 
AGARWAL, R.L. Seed technology. Oxford and IBH Publishing, 2018.

AHSANULLAH, M. et al. Distribution. In: Normal and student's distributions and their applications. Atlantis Press, 2014.

ALVES, R.M. et al. Stored diaspores of Astronium urundeuva Fr. (M. Allemão) Engl. (Anacardiaceae) submitted to hydropriming. Journal of Seed Science, v.42, e202042026, 2020.

ANDRADE, T.C.S. et al. Is the physiological potential of oilseed rape influenced by fertilization with nitrogen and sulfur? Journal of Seed Science, v.42, e202042027, 2020.

BANZATO, D.A.; KRONKA, S.N. Experimentação agrícola. 4.ed. Jaboticabal: FUNEP, 2006.

BAZZO, J.H.S. et al. Sowing dates and densities on physiological potential of seeds of white oat cultivars. Journal of Seed Science, v.42, e202042023, 2020.

BELNIAKI, A.C.E. et al. Rapid results of peach palm seed viability: a methodological proposition for the tetrazolium test. Journal of Seed Science, v.42, e202042034, 2020.

BEM JUNIOR, L.D. et al. Impact of storage on the physiological quality of soybean seeds after treatment with fungicides and insecticides. Journal of Seed Science, v.42, e202042037, 2020.

BENECH-ARNOLD, R. et al. Handbook of seed physiology: Applications to agriculture. CRC Press, 2004.

BRASIL. Instrução para a Análise de Sementes de Espécies Florestais. Ministério da Agricultura, Pecuária e Abastecimento. Secretaria de Defesa Agropecuária. Brasília: Mapa/ACS, 2013.

BRASIL. Manual de Análise de Sementes. Ministério da Agricultura, Pecuária e Abastecimento. Secretaria de Defesa Agropecuária. Brasília: Mapa/ACS, 2009.

CARVALHO, N.M.; NAKAGAWA, J. Sementes: ciência, tecnologia e produção. 5.ed. Jaboticabal: FUNEP, 2012.

CARVALHO, E.R. et al. Phytotoxicity in soybean seeds treated with phytosanitary products at different application times. Journal of Seed Science, v.42, e202042036, 2020.

CASTAN, D.O.C. et al. Vigor-S, a new system for evaluating the physiological potential of maize seeds. Scientia Agricola, v.75, n.2, p.167-172, 2018.

CORREA, A.R. et al. Germination and seed ecology of Buchenavia tomentosa Eichler (Combretaceae). Journal of Seed Science, v.42, 2020.

FERREIRA, A.G.; BORGHETTI, F. Germinação do básico ao aplicado. Porto Alegre: Artmed, 2004.

GAZOLA, S. et al. Probit regression to estimate the physiological potential of hybrid maize seed. Journal of Seed Science, v.37, n.1, p.33-39, 2015

GU, R. et al. Effect of mechanical threshing on damage and vigor of maize seed threshed at different moisture contents. Journal of Integrative Agriculture, v.18, n.7, p.1571-1578, 2019.

KHARE, D.; BHALE, M. Seed technology. Scientific Publishers, 2016.

KRZYZANOWSKI, F.C. et al. Physiological and sanitary performance of soybean seeds during storage after phosphine fumigation. Journal of Seed Science, v.41, n.3, p.280-285, 2019.

MACHADO, J.C. Tratamento de sementes no controle de doenças. Lavras: LAPS/FAEPE, 2000.

MAHDAVI, A.; KUNDU, D. A new method for generating distributions with an application to exponential distribution. Communications in Statistics-Theory and Methods, v.46, n.13, p.6543-6557, 2017.

MEDEIROS, J.A.D. et al. Vigor test of (strong) normal intact Amburana cearensis (Allemão) A.C. Smith seedlings. Journal of Seed Science, v.42, e202042011, 2020.

MERCADO, S.A.S. et al. Improvement of the methodology of the tetrazolium test using different pretreatments in seeds of the genus Epidendrum (Orchidaceae). Journal of Seed Science, v.42, e202042013, 2020.

MORAIS, T.C. et al. Physiological and antioxidant changes in sunflower seeds under water restriction. Journal of Seed Science, v.42, e202042008, 2020.

NELDER, J.A.; WEDDERBURN, R.M.W. Generalized linear models. Journal of the Royal Statistical Society: Series A (General), v.135, n.3, p.370-384, 1972.

OLIVEIRA, A.M.S. et al. Accelerated aging for evaluation of vigor in Brachiaria brizantha 'Xaraés' seeds. Journal of Seed Science, v.42, e202042006, 2020.

OROBINSKY, V.I. et al. Seed refinement in the harvesting and post-harvesting process. In: International scientific and practical conference Agro-SMART-Smart solutions for agriculture. Atlantis 
Press, 2018

PINHEIRO, D.T. et al. Antioxidant enzyme activity and physiological potential of Capsicum baccatum var. baccatum seeds as a function of post-harvest storage of fruit. Journal of Seed Science, v.42, e202042028, 2020.

PINTO, M.B.A. et al. Productivity and physiological quality of seeds with burn down herbicides at the pre harvest of bean crops. Journal of Seed Science, v.36, n.4, p.384-391,2014.

PRADO, J.P. et al. Physiological potential of soybean seeds and its relationship to electrical conductivity. Journal of Seed Science, v.41, n.4, p.407-415, 2019

RIFNA, E.J. et al. Emerging technology applications for improving seed germination. Trends in Food Science \& Technology, v.86, p.95-108, 2019.

RUDNEV, S.G. Principles of organization of post-harvest grain processing. In: International Scientific and Practical Conference World science. ROST, 2017.

SANTANA, D.G. et al. How to analyze germination of species with empty seeds using contemporary statistical methods? Acta Botanica Brasilica, n.1, p.271-278, 2018.

SCOTT, S.J. et al. Review of Data Analysis Methods for Seed Germination. Cropscience, v.4, n.6, p.1192-1199, 1984.

SILVA, J.N. et al. Cowpea yield and quality after application of desiccating herbicides. Journal of Seed Science, V.42, e202042019, 2020

SOARES, T.F.S.N. et al. Assessment of seed vigor tests for Crambe abyssinica. Journal of Agricultural Science, v.10, n.12, p.527-533, 2018.

SOUZA, V.C. et al. Vigor de sementes armazenadas de ipêamarelo Tabebuia serratifolia (Vahl.) Nich. Revista Árvore, v.29, n.6, p.833-841, 2005

WIJEWARDANA, C. et al. Soybean seed physiology, quality, and chemical composition under soil moisture stress. Food chemistry, v.278, p.92-100, 2019. 